\title{
A COMPARISON OF REDUCED-ORDER MODELING APPROACHES USING ARTIFICIAL NEURAL NETWORKS FOR PDES WITH BIFURCATING SOLUTIONS*
}

\author{
MARTIN W. HESS ${ }^{\dagger}$, ANNALISA QUAINI $^{\ddagger}$, AND GIANLUIGI ROZZA ${ }^{\dagger}$
}

\begin{abstract}
This paper focuses on reduced-order models (ROMs) built for the efficient treatment of PDEs having solutions that bifurcate as the values of multiple input parameters change. First, we consider a method called local ROM that uses k-means algorithm to cluster snapshots and construct local POD bases, one for each cluster. We investigate one key ingredient of this approach: the local basis selection criterion. Several criteria are compared and it is found that a criterion based on a regression artificial neural network (ANN) provides the most accurate results for a channel flow problem exhibiting a supercritical pitchfork bifurcation. The same benchmark test is then used to compare the local ROM approach with the regression ANN selection criterion to an established global projection-based ROM and a recently proposed ANN based method called POD-NN. We show that our local ROM approach gains more than an order of magnitude in accuracy over the global projection-based ROM. However, the POD-NN provides consistently more accurate approximations than the local projection-based ROM.
\end{abstract}

Key words. Navier-Stokes equations, reduced-order methods, reduced basis methods, parametric geometries, symmetry breaking bifurcation

AMS subject classifications. 65P30, 35B32, 35Q30, 65N30, 65N35, 65N99

1. Introduction. We consider the problem of finding a function $u \in V$ such that

$$
\mathcal{N}(u, v ; \boldsymbol{\mu})=\mathcal{F}(v ; \boldsymbol{\mu}) \quad \forall v \in V,
$$

where $\boldsymbol{\mu} \in D$ denotes a point in a parameter domain $D \subset \mathbb{R}^{M}, V$ a function space, $\mathcal{N}(\cdot, \cdot ; \boldsymbol{\mu})$ a given form that is linear in $v$ but generally nonlinear in $u$, and $\mathcal{F}(\cdot)$ a linear functional on $V$. Note that either $\mathcal{N}$ or $\mathcal{F}$ or both could depend on some or all the components of the parameter vector $\boldsymbol{\mu}$. We view the problem (1.1) as a variational formulation of a nonlinear partial differential equation (PDE) or a system of such equations in which $M$ parameters appear. We are interested in systems that undergo bifurcations, i.e., the solution $u$ of (1.1) differs in character for parameter vectors $\boldsymbol{\mu}$ belonging to different subregions of $D$. We are particularly interested in situations that require solutions of (1.1) for a set of parameter vectors that span across two or more of the subregions of the bifurcation diagram. This is the case, for example, if one needs to trace the bifurcation diagram.

In general, one could approximate the solutions to (1.1) using a Full Order Method (FOM), like for example the Finite Element Method or the Spectral Element Method. Let $V_{N}$ be a $N$-dimensional subspace that is a subset of $V$. A FOM seeks an approximation $u_{N} \in V_{N}$ such that

$$
\mathcal{N}_{h}\left(u_{N}, v ; \boldsymbol{\mu}\right)=\mathcal{F}_{h}(v ; \boldsymbol{\mu}) \quad \forall v \in V_{N},
$$

*Received September 30, 2020. Accepted June 09, 2021. Published online on December 17, 2021. Recommended by Peter Benner. We acknowledge the support by European Union Funding for Research and Innovation - Horizon 2020 Program - in theframework of European Research Council Executive Agency: Consolidator Grant H2020 ERC CoG 2015 AROMA-CFD project 681447 "Advanced Reduced Order Methods with Applications in Computational Fluid Dynamics" (P.I. Prof. Gianluigi Rozza). We also acknowledge the INDAM-GNCS project "Advanced intrusive and non-intrusive model order reduction techniques and applications", 2019. We acknowledge project MIUR PRIN 2017 NA-FROM-PDEs. This work was also partially supported by US National Science Foundation through grant DMS-1620384 and DMS-195353.

†SISSA Mathematics Area, mathLab, International School for Advanced Studies, via Bonomea 265, I-34136 Trieste, Italy ( $\{$ mhess, grozza\}@sissa.it).

${ }^{\ddagger}$ Department of Mathematics, University of Houston, Houston, Texas 77204, USA (qua ini@math. uh. edu). 
where $\mathcal{N}_{h}$ and $\mathcal{F}_{h}$ are the discretized forms for $\mathcal{N}$ and $\mathcal{F}$. FOMs are often expensive, especially if multiple solutions are needed. For this reason, one is interested in finding surrogate methods that are much less costly. Such surrogates are constructed using a "few" solutions obtained with the FOM. Here, we are interested in reduced-order models (ROMs) for which one constructs a low-dimensional approximating subspace $V_{L} \subset V_{N}$ of dimension $L$ that still contains an acceptably accurate approximation $u_{L}$ to the FOM solution $u_{N}$, and thus also to the solution $u$ of (1.1). That approximation is determined from the reduced discrete system

$$
\mathcal{N}_{h}\left(u_{L}, v ; \boldsymbol{\mu}\right)=\mathcal{F}_{h}(v ; \boldsymbol{\mu}) \quad \forall v_{L} \in V_{L}
$$

that, if $L \ll N$, is much cheaper to solve compared to (1.2). We refer to approach (1.3) as global ROM, since a single global basis is used to determine the ROM approximation $u_{L}$ at any chosen parameter point $\boldsymbol{\mu} \in D$.

Global ROMs in the setting of bifurcating solutions are considered in the early papers [15, $16,17,18]$ for buckling bifurcations in solid mechanics. More recently, in [26] it is shown that a Proper Orthogonal Decomposition (POD) approach allows for considerable computational time savings for the analysis of bifurcations in some nonlinear dissipative systems. Reduced Basis (RB) methods have been used to study symmetry breaking bifurcations [6, 24] and Hopf bifurcations [23] for natural convection problems. A RB method for symmetry breaking bifurcations in contraction-expansion channels has been proposed in [22]. A RB method for the stability of flows under perturbations in the forcing term or in the boundary conditions, is introduced in [27]. Furthermore, in [27] it is shown how a space-time inf-sup constant approaches zero as the computed solutions get close to a bifurcating value. Recent works have proposed ROMs for bifurcating solutions in structural mechanics [20] and for a nonlinear Schrödinger equation, called Gross-Pitaevskii equation [19], respectively. Finally, we would like to mention that machine learning techniques based on sparse optimization have been applied to detect bifurcating branches of solutions for a two-dimensional laterally heated cavity and Ginzburg-Landau model in [2, 12], respectively. Finding all branches after a bifurcation occurs can be done with deflation methods (see [21]), which require introducing a pole at each known solution. In principle, the ROM approaches under investigation here can be combined with deflation techniques.

In a setting of a bifurcation problem (i.e., $D$ consists of subregions for which the corresponding solutions of (1.1) have different character), it may be the case that $L$, although small compared to $N$, may be large enough so that solving system (1.3) many times becomes expensive. To overcome this problem, in [7] we proposed a local ROM approach. The idea is to construct several local bases (in the sense that they use solutions for parameters that lie in subregions of the parameter domain), each of which is used for parameters belonging to a different subregion of the bifurcation diagram. So, we construct $K$ such local bases of dimension $L_{k}$, each spanning a local subspace $V_{L_{k}} \subset V_{N}$. We then construct $K$ local reduced-order models

$$
\mathcal{N}\left(u_{L_{k}}, v ; \boldsymbol{\mu}\right)=\mathcal{F}(v ; \boldsymbol{\mu}) \quad \forall v \in V_{L_{k}} \quad \text { for } k=1, \ldots, K
$$

that provide acceptably accurate approximations $u_{L_{k}}$ to the solution $u$ of (1.1) for parameters $\boldsymbol{\mu}$ belonging to different parts of the bifurcation diagram. A key ingredient in this approach is how to identify which local basis should be used in (1.4) to determine the corresponding ROM approximation for any parameter point $\boldsymbol{\mu} \in D$ that was not among those used to generate the snapshots. Several criteria are proposed and compared for a two-parameter study in Section 3.1. To the best of our knowledge, no work other than [7] addresses the use of local ROM basis for bifurcation problems. This is the continuation of previous work on model reduction with spectral element methods [10] and including parametric variations of the geometry [8,9]. 
This paper aims at comparing one global ROM approach, our local ROM approach with the "best" criterion to select the local basis, and a recently proposed RB method that uses neural networks to accurately approximate the coefficients of the reduced model [11]. This third method is referred to as POD-NN. The global ROM as explained in, e.g., uses the most dominant POD modes of a uniform snapshot set over the parameter domain. The dominant modes define the projection space for every parameter evaluation of interest and is in this sense global with respect to the parameter space. See, e.g., [13] for more details. Like the global ROM, the POD-NN employs the most dominant POD modes. However, the difference is that the coefficients of the snapshots in the ROM expansion are used as training data for an artificial neural network. The local ROM first employs a classification ANN to determine the corresponding cluster of a parameter location, but this can be improved upon with a regression ANN using the relative errors of the local ROMs at the snapshot locations as training data. As a concrete setting for the comparison, we use the Navier-Stokes equations and in particular flow through a channel with a contraction.

The outline of the paper is as follows. In Section 2, we briefly present the Navier-Stokes equations and consider a specific benchmark test. Section 3 reports the comparison of many local basis selection criteria for the local ROM approach and the main comparison of the three ROM approaches. Concluding remarks are provided in Section 4.

2. Application to the incompressible Navier-Stokes equations. The Navier-Stokes equations describe the incompressible motion of a viscous, Newtonian fluid in the spatial domain $\Omega \subset \mathbb{R}^{d}, d=2$ or 3 , over a time interval of interest $(0, T]$. They are given by

$$
\begin{aligned}
\frac{\partial \boldsymbol{u}}{\partial t}+(\boldsymbol{u} \cdot \nabla \boldsymbol{u})-\nu \Delta \boldsymbol{u}+\nabla p & =\mathbf{0} & & \text { in } \Omega \times(0, T] \\
\nabla \cdot \boldsymbol{u} & =0 & & \text { in } \Omega \times(0, T],
\end{aligned}
$$

where $\boldsymbol{u}$ and $p$ denote the unknown velocity and pressure fields, respectively, and $\nu>0$ denotes the kinematic viscosity of the fluid. Note that there is no external body force because we will not need one for the specific benchmark test under consideration.

Problem (2.1) needs to be endowed with initial and boundary conditions, e.g.:

$$
\begin{aligned}
\boldsymbol{u}=\boldsymbol{u}_{0} & \text { in } \Omega \times\{0\} \\
\boldsymbol{u}=\boldsymbol{u}_{D} & \text { on } \partial \Omega_{D} \times(0, T] \\
-p \boldsymbol{n}+\nu \frac{\partial \boldsymbol{u}}{\partial \boldsymbol{n}}=\boldsymbol{g} & \text { on } \partial \Omega_{N} \times,(0, T]
\end{aligned}
$$

where $\partial \Omega_{D} \cap \partial \Omega_{N}=\emptyset$ and $\overline{\partial \Omega_{D}} \cup \overline{\partial \Omega_{N}}=\overline{\partial \Omega}$. Here, $\boldsymbol{u}_{0}, \boldsymbol{u}_{D}$, and $\boldsymbol{g}$ are given and $\boldsymbol{n}$ denotes the unit normal vector on the boundary $\partial \Omega_{N}$ directed outwards. In the rest of this section, we will explicitly denote the dependence of the solution of the problem (2.1)-(2.4) on the parameter vector $\boldsymbol{\mu}$.

Let $L^{2}(\Omega)$ denote the space of square integrable functions in $\Omega$ and $H^{1}(\Omega)$ the space of functions belonging to $L^{2}(\Omega)$ with first derivatives in $L^{2}(\Omega)$. Moreover, let

$$
\begin{aligned}
\boldsymbol{V} & :=\left\{\boldsymbol{v} \in\left[H^{1}(\Omega)\right]^{d}: \boldsymbol{v}=\boldsymbol{u}_{D} \text { on } \partial \Omega_{D}\right\}, \\
\boldsymbol{V}_{0} & :=\left\{\boldsymbol{v} \in\left[H^{1}(\Omega)\right]^{d}: \boldsymbol{v}=\mathbf{0} \text { on } \partial \Omega_{D}\right\} .
\end{aligned}
$$


The standard variational form corresponding to (2.1)-(2.4) is: find $(\boldsymbol{u}(\boldsymbol{\mu}), p(\boldsymbol{\mu})) \in \boldsymbol{V} \times L^{2}(\Omega)$ satisfying the initial condition (2.2) and

$$
\begin{gathered}
\int_{\Omega} \frac{\partial \boldsymbol{u}(\boldsymbol{\mu})}{\partial t} \cdot \boldsymbol{v} \mathrm{d} \mathbf{x}+\int_{\Omega}(\boldsymbol{u}(\boldsymbol{\mu}) \cdot \nabla \boldsymbol{u}) \cdot \boldsymbol{v} \mathrm{d} \mathbf{x}-\int_{\Omega} p(\boldsymbol{\mu}) \nabla \cdot \boldsymbol{v} \mathrm{d} \mathbf{x} \\
=\int_{\partial \Omega_{N}} \mathbf{g} \cdot \boldsymbol{v} \mathrm{d} \mathbf{x}, \quad \forall \boldsymbol{v} \in \boldsymbol{V}_{0} \\
\int_{\Omega} q \nabla \cdot \boldsymbol{u}(\boldsymbol{\mu}) \mathrm{d} \mathbf{x}=0, \quad \forall q \in L^{2}(\Omega) .
\end{gathered}
$$

Problem (2.5) constitutes the particular case of the abstract problem (1.1) we use for the numerical illustrations.

We consider a benchmark test that has been widely studied in the literature: channel flow through a narrowing of width $w$; see, e.g., $[3,4,5,14]$ and the references cited therein. The 2D geometry under consideration is depicted in Figure 2.1. A parabolic horizontal velocity component with maximum $\frac{9}{4}$ and zero vertical component is inscribed on the inlet at the left side. At the top and bottom of the channel as well as the narrowing boundaries, zero velocity walls are assumed. The right end of the channel is an outlet, where zero Neumann boundaries (i.e., $\mathbf{g}=\mathbf{0}$ ) are assumed. We will let both the narrowing width and the viscosity vary in given ranges that include a bifurcation.
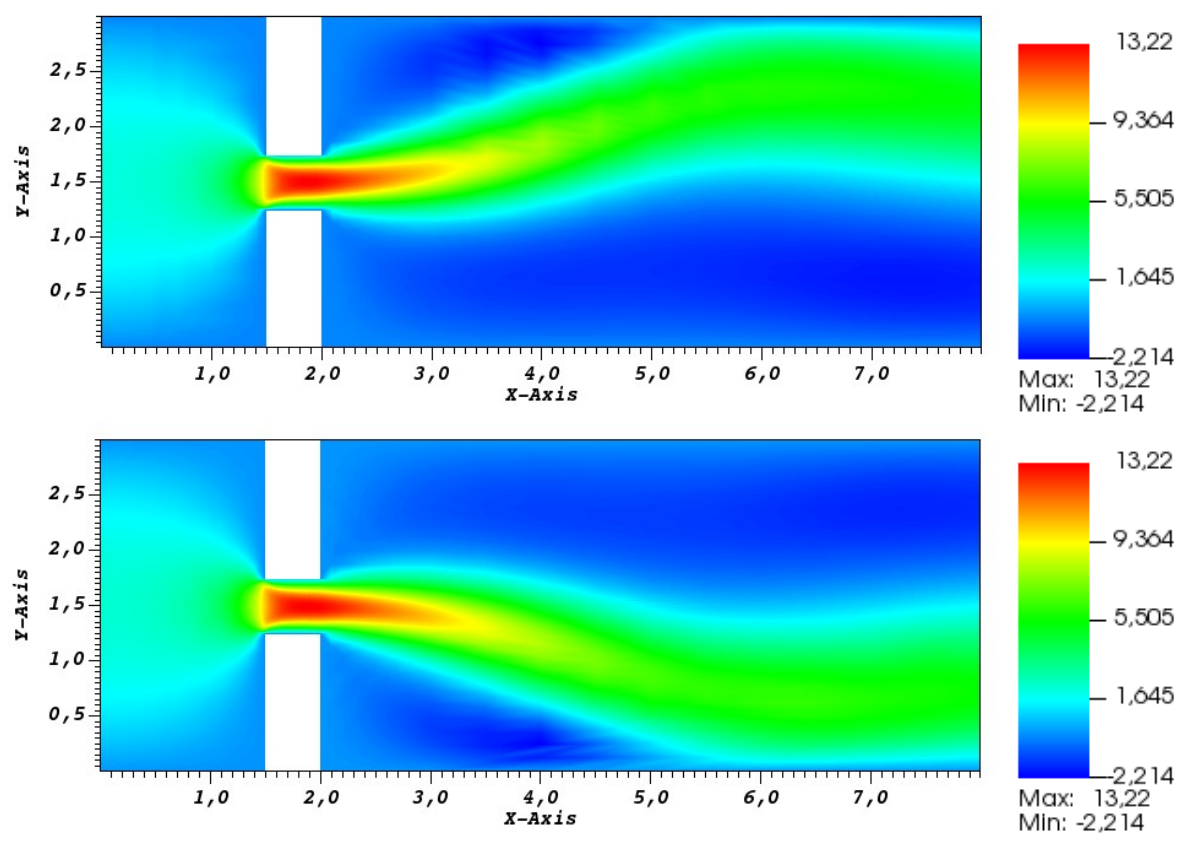

FIG. 2.1. Steady state solutions for kinematic viscosity $\nu=0.1$ and orifice width $w=0.5$. Shown is the horizontal component of the velocity. The two stable solutions can be characterized by an attachment to the upper and lower wall, respectively.

The Reynolds number Re can be used to characterize the flow regime. For the chosen data, we have $\operatorname{Re}=9 /(4 \nu)$, since the characteristic length has been set to one. As the Reynolds number Re increases from zero, we first observe a steady symmetric jet with two 
recirculation regions downstream of the narrowing that are symmetric about the centerline. As $\mathrm{Re}$ increases, the recirculation length progressively increases. At a certain critical value $\mathrm{Re}_{\text {crit }}$, one recirculation zone expands whereas the other shrinks, giving rise to a steady asymmetric jet. This asymmetric solution remains stable as Re increase further, but the asymmetry becomes more pronounced. The configuration with a symmetric jet is still a solution, but is unstable [25]. Snapshots of the stable solutions for kinematic viscosity $\nu=0.1$ and orifice width $w=0.5$ are illustrated in Figure 2.1. This loss of symmetry in the steady solution as Re changes is a supercritical pitchfork bifurcation [1].

Because we are interested in studying a flow problem close to a steady bifurcation point, our snapshot sets include only steady-state solutions [7]. To obtain the snapshots, we approximate the solution of problem (2.5) by a time-marching scheme that we stop when sufficiently close to the steady state, e.g., when the stopping condition

$$
\frac{\left\|\boldsymbol{u}_{N}^{n}-\boldsymbol{u}_{N}^{n-1}\right\|_{L^{2}(\Omega)}}{\left\|\boldsymbol{u}_{N}^{n}\right\|_{L^{2}(\Omega)}}<\text { tol }
$$

is satisfied for a prescribed tolerance tol $>0$, where $n$ denotes the time-step index.

3. Numerical results. We conduct a parametric study for the channel flow where we let the the viscosity $\nu$ (physical parameter) vary in $[0.1,0.2]$ and the narrowing width $w$ (geometric parameter) vary in $[0.5,1.0]$.

We choose the Spectral Element Method (SEM) as FOM. For the spectral element discretization, the SEM software framework Nektar++, version 4.4.0, (see https: / / www . nektar.info/) is used. The domain is discretized into 36 triangular elements as shown in Figure 3.1. Modal Legendre ansatz functions of order 12 are used in every element and for every solution component. This results in 4752 degrees of freedom for each of the horizontal and vertical velocity components and the pressure for the time-dependent simulations. For temporal discretization, an IMEX scheme of order 2 is used with a time-step size of $\Delta t=10^{-4}$; typically $10^{5}$ time steps are needed to reach a steady state.

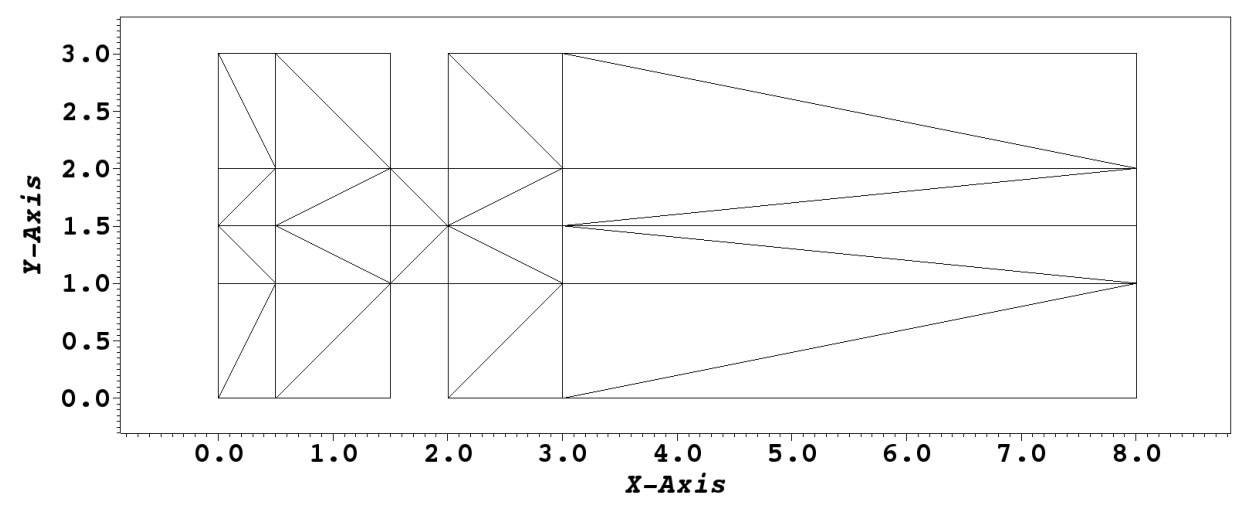

FIG. 3.1. The 36 triangular elements used for spatial approximation.

Figure 3.2 shows a bifurcation diagram: the vertical component of the velocity at the point $(3.0,1.5)$ computed by SEM is plotted over the parameter domain. The reference snapshots have been computed on a uniform $40 \times 41$ grid. Notice that Figure 3.2 reports only the lower branch of asymmetric solutions.

In [7], we presented preliminary results obtained with our local ROM approach for a two-parameter study related to the channel flow. We showed that two criteria to select the 


\section{ETNA}

Kent State University and

Johann Radon Institute (RICAM)

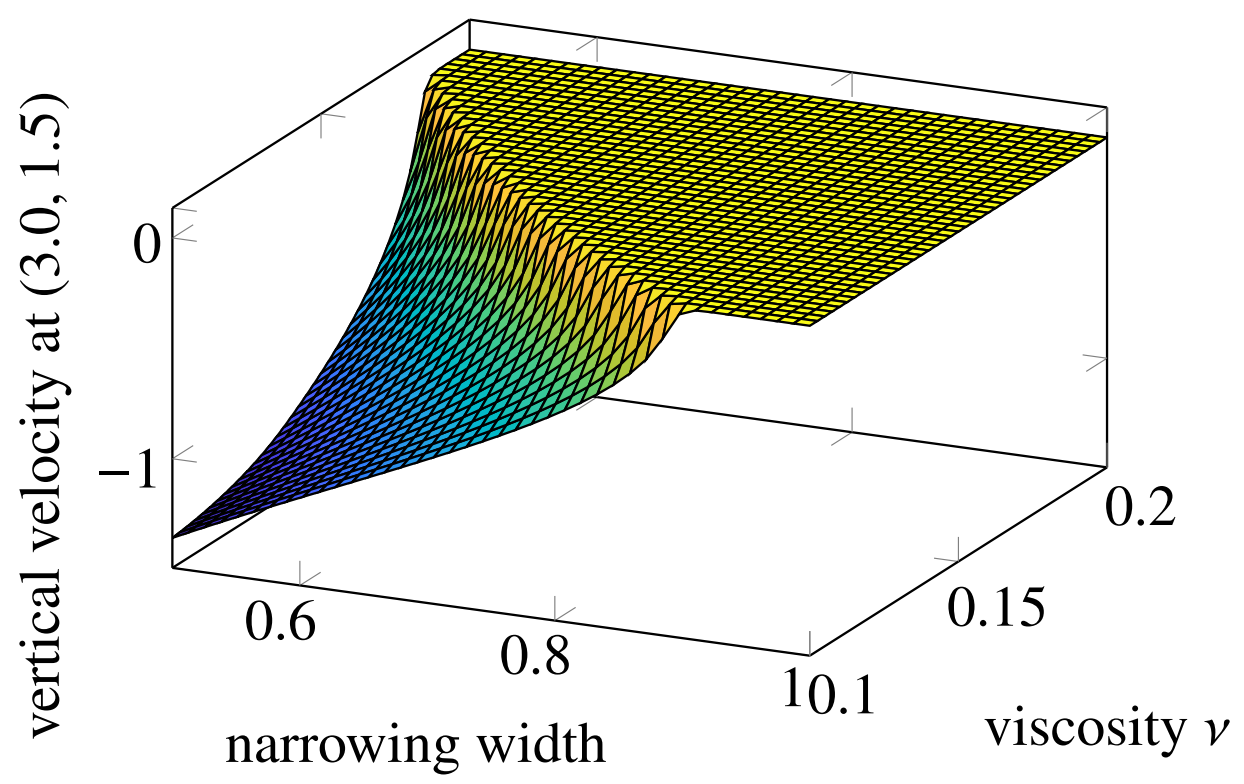

FIG. 3.2. Bifurcation diagram of the channel with variable width and viscosity: vertical component of the velocity computed by SEM close to steady state and evaluated at the point $(3.0,1.5)$. Color encodes the vertical velocity component.

local ROM basis that work well for one-parameter studies fail for the two-parameter case, in the sense that they provide a poor reconstruction of the bifurcation diagram. Section 3.1 addresses the need to find an accurate and inexpensive criterion to assign the local ROM basis for a given parameter $\boldsymbol{\mu}$ in a multi-parameter context.

The comparison for global ROM, local ROM, and POD-NN is reported in Section 3.2.

3.1. Comparing criteria to select the local basis in the local ROM approach. For our local ROM approach, we sample 72 snapshots and divide them into 8 clusters using k-means clustering. The number of clusters is chosen according to the minimal k-means energy. For more details, we refer to [7].

First, we consider the two criteria presented in [7], namely the distance to parameter centroid and the distance to the closest snapshot location. The first criterion entails finding the closest parameter centroid and using the corresponding local ROM basis. The second criterion finds the closest snapshot location to the given parameter vector $\boldsymbol{\mu}$ and the local ROM basis that includes this snapshot is considered. The bifurcation diagrams reconstructed by the local ROM approach with these two criteria are compared in Figure 3.3. The distance to parameter centroid criterion does not manage to recover the bifurcation diagram well and this seems largely due to the local ROM assignment scheme; see Figure 3.3 (top). In the bifurcation diagram corresponding to the distance to the closest snapshot location, we observe several jumps when moving from one cluster to the next; see Figure 3.3 (bottom). These jumps, which correspond to large approximation errors, can perhaps be better appreciated from another view of the same bifurcation diagram shown in Figure 3.4. Next, we will try to reduce the jumps.

To alleviate the bad approximation in the transition regions between two clusters (i.e., local ROMs), we introduce overlapping clusters. In particular, the collected snapshots of a cluster from the k-means algorithm undergo a first POD and are then enriched with the orthogonal complement of neighboring snapshots according to the sampling grid. Then, a second POD with a lower POD tolerance defines the ROM ansatz space. The corresponding 


\section{ETNA}

Kent State University and

Johann Radon Institute (RICAM)
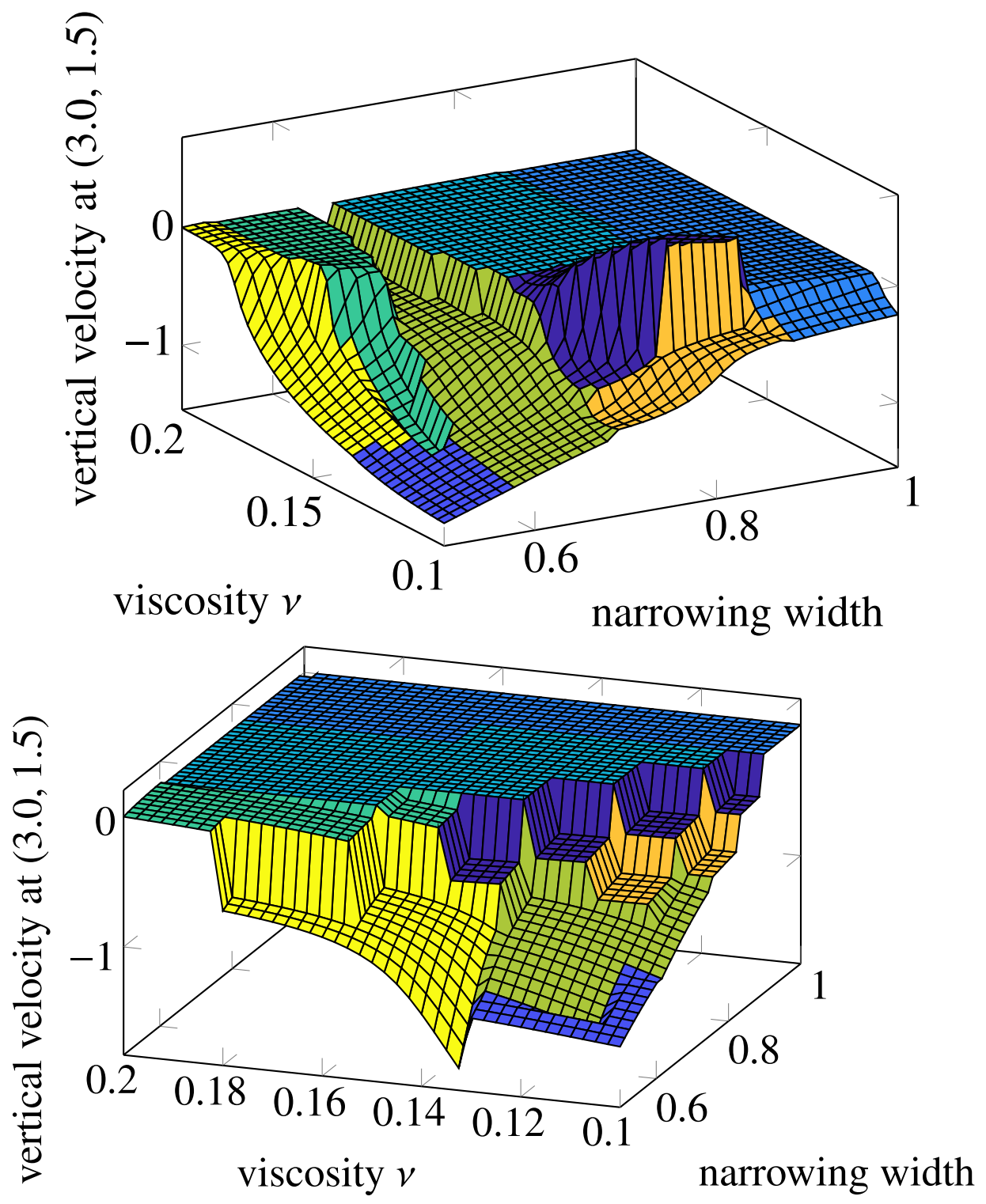

FIG. 3.3. Local ROM: bifurcation diagram reconstructed with basis selection criterion distance to parameter centroid (top) and distance to the closest snapshot location (bottom). Different colors are used for different clusters.

bifurcation diagram is shown in Figure 3.5. We observe that several jumps have been smoothed out; compare Figure 3.5 (top) with Figure 3.4. The mean approximation error reduces by about an order of magnitude thanks to the overlap.

Although the overlapping clusters lead to a better reconstruction of the bifurcation diagram, the result is still not satisfactory. Thus, we propose an alternative selection criterion that uses an artificial neural network (ANN). The ANN is trained using the k-means clustering as training information and enforcing a perfect match at the snapshot locations with the corresponding cluster. This is a classification problem, implemented in Keras Tensorflow. The ANN is designed as multilayer perceptron with 4 layers, the first layer having just two nodes (or 


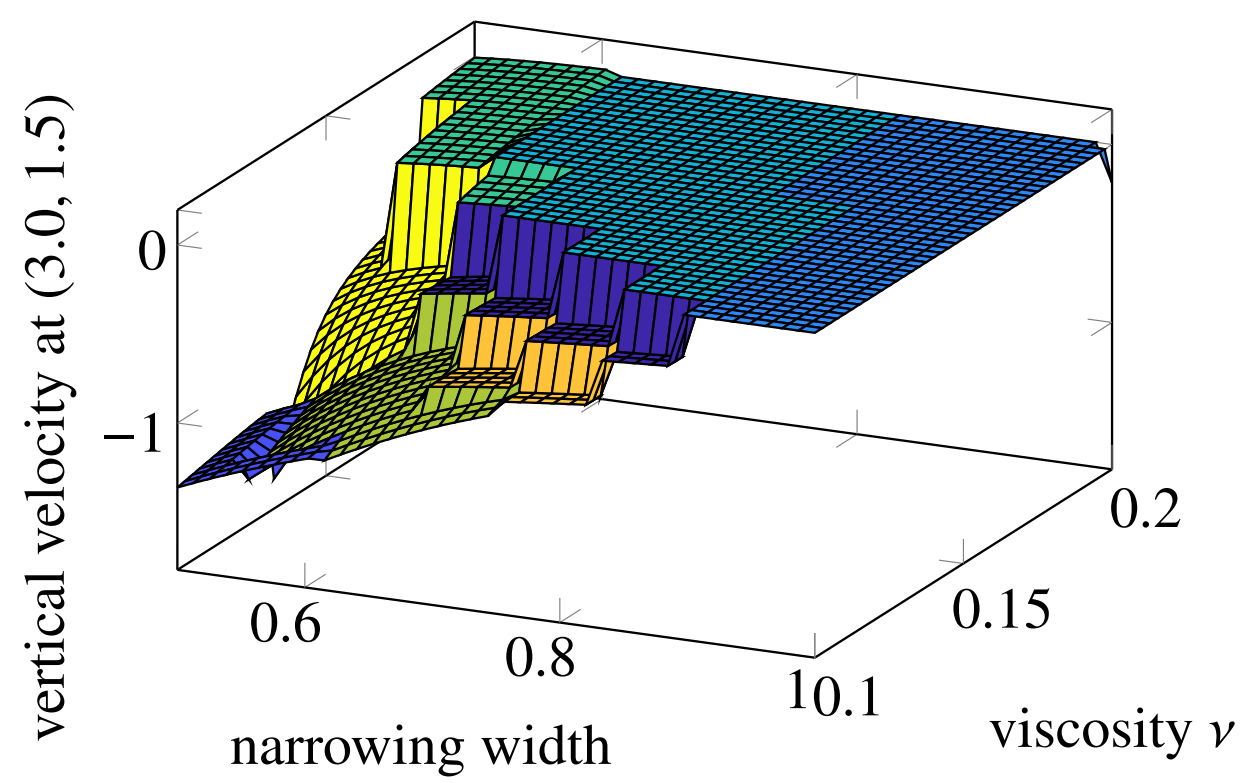

FIG. 3.4. Different view of the bifurcation diagram shown in Figure 3.3 (bottom).

"neurons") taking the two dimensional parameter values. The two inner layers are big (2048 and 1024 nodes, respectively), while the last layer corresponds to the number of clusters, so 8 in this case. For the first three layers a ReLU activation function is used, while for the last layer activation function is a softmax. The perfect match can be enforced by either running the training as long as the training data can be exactly matched or using Keras early stopping with an outer loop checking for a match.

Figure 3.6 shows the bifurcation diagram reconstructed with the ANN selection criterion. We observe a better reconstruction of the bifurcation diagram; compare Figure 3.6 with Figure 3.3 and 3.5.

For a more quantitative comparison, we run 4 tests. The tests differ in the number of samples and whether cluster overlapping is used. The specifications of each test are reported in Tables 3.1 and 3.2, which list the relative errors evaluated over the $40 \times 41$ reference grid in the $L^{2}$ and $L^{\infty}$ norms, respectively. Three local basis selection criteria are considered: distance to parameter centroid, distance to the closest snapshot location, and ANN. Tables 3.1 and 3.2 confirm that the overlapping cluster represent an improvement over non-overlapping clusters. This is true for all three criteria, but in particular for the distance to parameter centroid criterion. Thus, for tests 3 and 4 we only used overlapping clusters. We notice that the ANN criterion outperforms the other two criteria in all the tests, both in the $L^{2}$ and $L^{\infty}$ norms. However, the margin of improvement becomes smaller as the number of samples increases. Tables 3.1 and 3.2 also report the mean relative error for an optimal cluster selection, which is explained next.

By "optimal cluster selection" we address the question of how parameter points are optimally associated with an already given clustering. Figure 3.7 shows the optimal cluster selection. This optimal selection can only be obtained from a fine grid of reference solutions. Thus it is usually not available. The reason why we report it is because some interesting conclusions can be drawn from it. First, the best possible clustering does not have contiguous clusters. This is in contrast to the clusters created by the k-means algorithm, which are contiguous in the parameter space in all of our tests. Second, the best possible approximation 

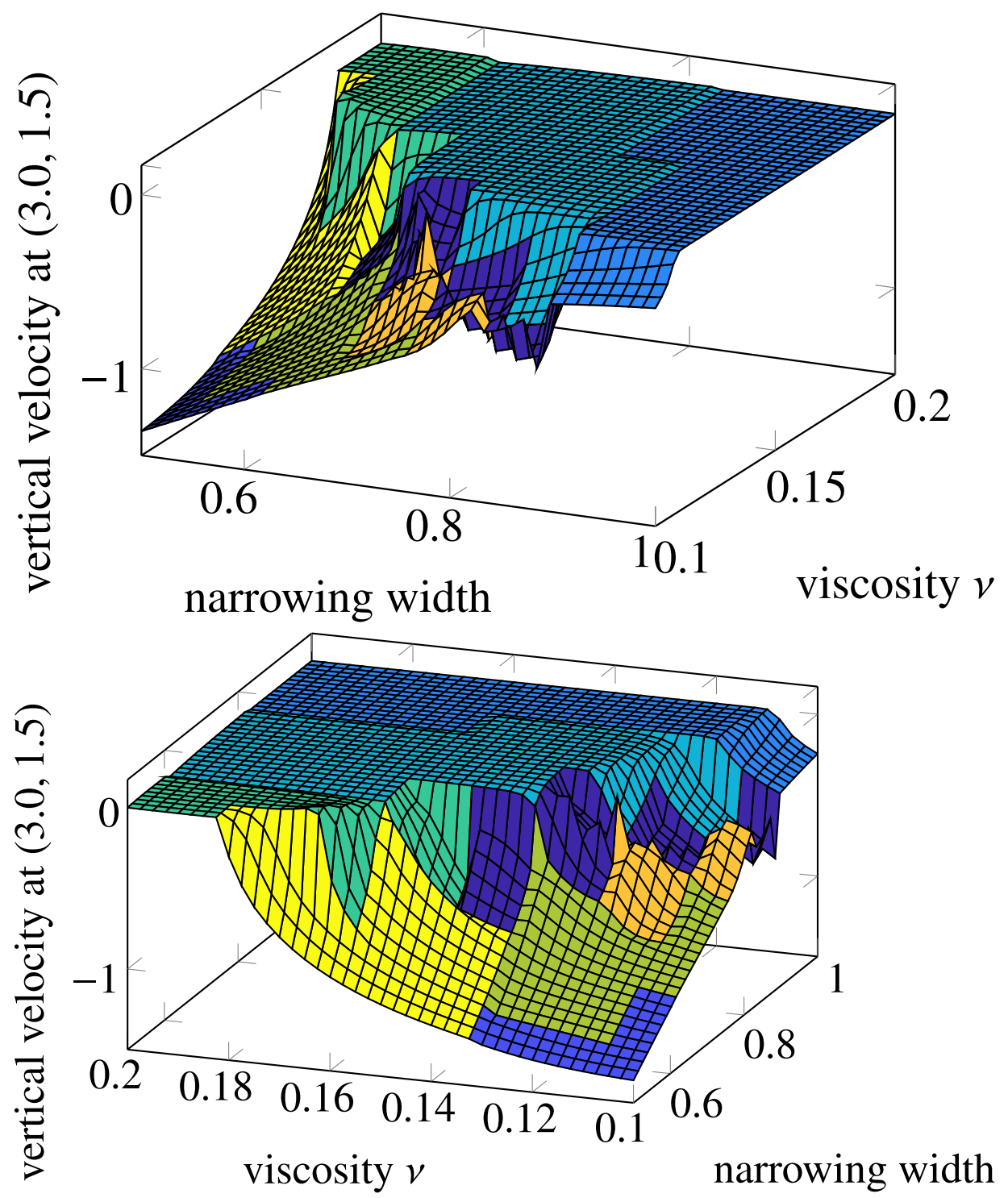

FIG. 3.5. Local ROM with basis selection criterion distance to the closest snapshot location and overlapping clusters: two views of the reconstructed bifurcation diagram. Different colors are used for different clusters.

at a snapshot location is not necessarily given by the local cluster to which that snapshot is assigned. Third, there still is a reduction factor of 5-20 in the relative $L^{2}$ for the velocity error that could be gained, as one can see when comparing the respective errors for the optimal cluster and the ANN selection criteria in Tables 3.1 and 3.2.

To get close to the optimum, we adopt the following strategy. We compute relative errors of all local ROMs at all snapshot locations. This operation is performed offline and is not expensive since the exact solution at the snapshot locations is available. The relative errors can be used as training data for an ANN, which means that the ANN training is treated as a regression and not a classification. Thus, the ANN will approximate relative errors of each local ROM over the parameter domain. This approximation is used as cluster selection 


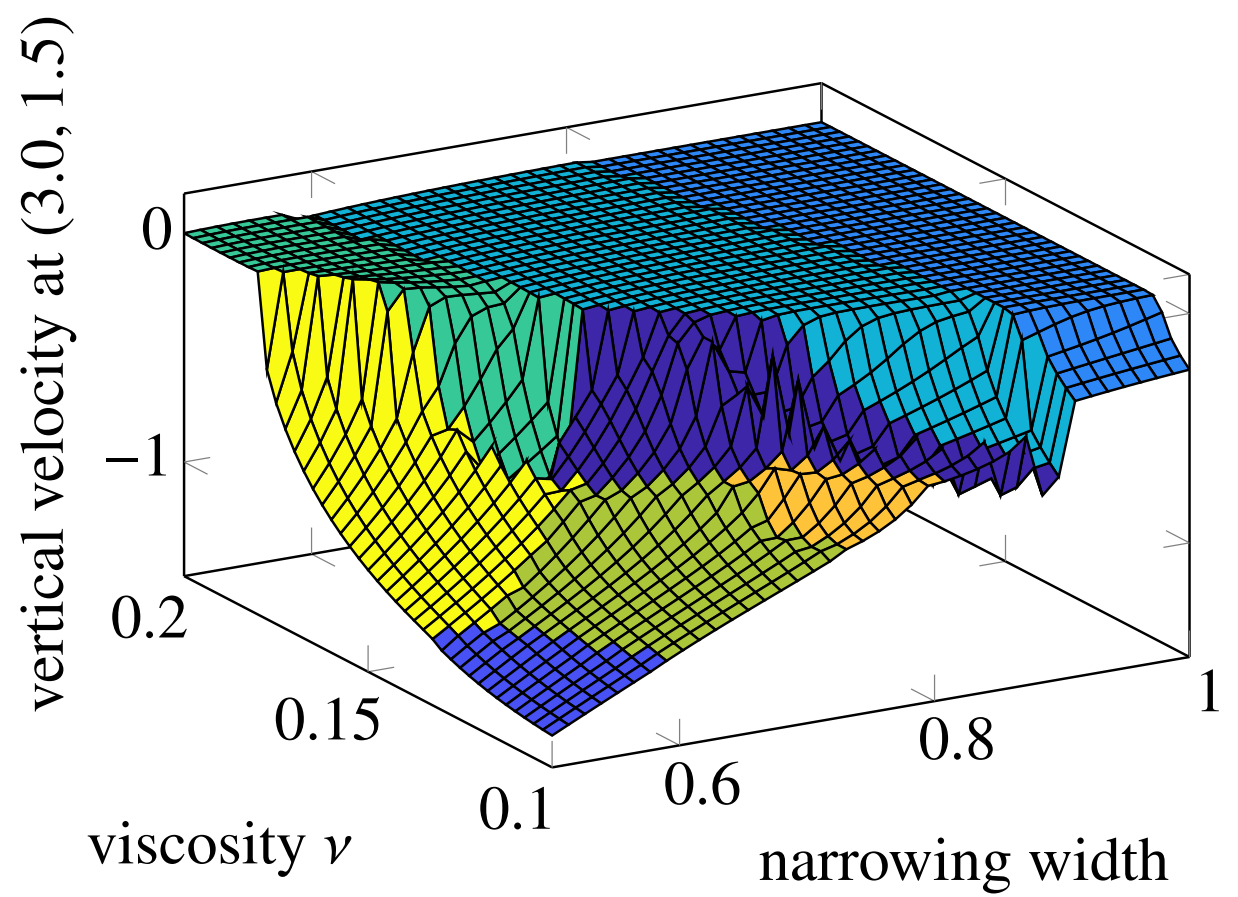

FIG. 3.6. Local ROM with basis selection selection that uses the ANN selection criterion: reconstruction of the bifurcation diagram.

TABLE 3.1

Local ROM with three different basis selection: mean relative $L^{2}$ errors for the velocity over all reference parameter points; 1640 on a uniform $40 \times 41$ grid.

\begin{tabular}{lcccc} 
& Test 1 & Test 2 & Test 3 & Test 4 \\
\hline samples & 72 & 72 & 110 & 240 \\
uniform grid & $8 \times 9$ & $8 \times 9$ & $10 \times 11$ & $15 \times 16$ \\
overlapping clusters & yes & no & yes & yes \\
parameter centroid mean & 0.0294 & 0.0632 & 0.1085 & 0.0559 \\
distance snapshot mean & 0.0241 & 0.0295 & 0.1011 & 0.0227 \\
ANN mean & 0.0238 & 0.0273 & 0.1002 & 0.0223 \\
optimum & 0.0046 & 0.0106 & 0.0048 & 0.0092
\end{tabular}

criterion. In this procedure, it is important to normalize the data. Here, we use the inverse relative error and normalize the error vectors at each snapshot location. We note that the training time for the ANN increases, but is still well below the ROM offline time, i.e., 30 minutes vs. several hours. The ROM offline time is dominated by computing the affine expansion of the trilinear form. The computational cost grows with the cube of the reduced order model dimension. This makes the localized ROM much faster than the global ROM as, for example, the global ROM might have dimension of 40 while each of the 8 local ROMs has a dimension of about 10 . We found it impossible to quantify the training cost of an ANN. The performance of the stochastic gradient employed in the ANN training varied significantly over multiple runs and required outer loops to check the accuracy. Thus, the given measure of 30 minutes vs. several hours is only our experience with this particular model and probably 


\section{ETNA}

Kent State University and

Johann Radon Institute (RICAM)

TABLE 3.2

Local ROM with three different basis selection: mean relative $L^{\infty}$ errors for the velocity over all reference parameter points; 1640 on a uniform $40 \times 41$ grid.

\begin{tabular}{lcccc} 
& Test 1 & Test 2 & Test 3 & Test 4 \\
\hline samples & 72 & 72 & 110 & 240 \\
uniform grid & $8 \times 9$ & $8 \times 9$ & $10 \times 11$ & $15 \times 16$ \\
overlapping clusters & yes & no & yes & yes \\
parameter centroid mean & 0.0275 & 0.0600 & 0.0970 & 0.0538 \\
distance snapshot mean & 0.0230 & 0.0284 & 0.0908 & 0.0217 \\
ANN mean & 0.0227 & 0.0263 & 0.0899 & 0.0214 \\
optimum & 0.0044 & 0.0101 & 0.0045 & 0.0088
\end{tabular}

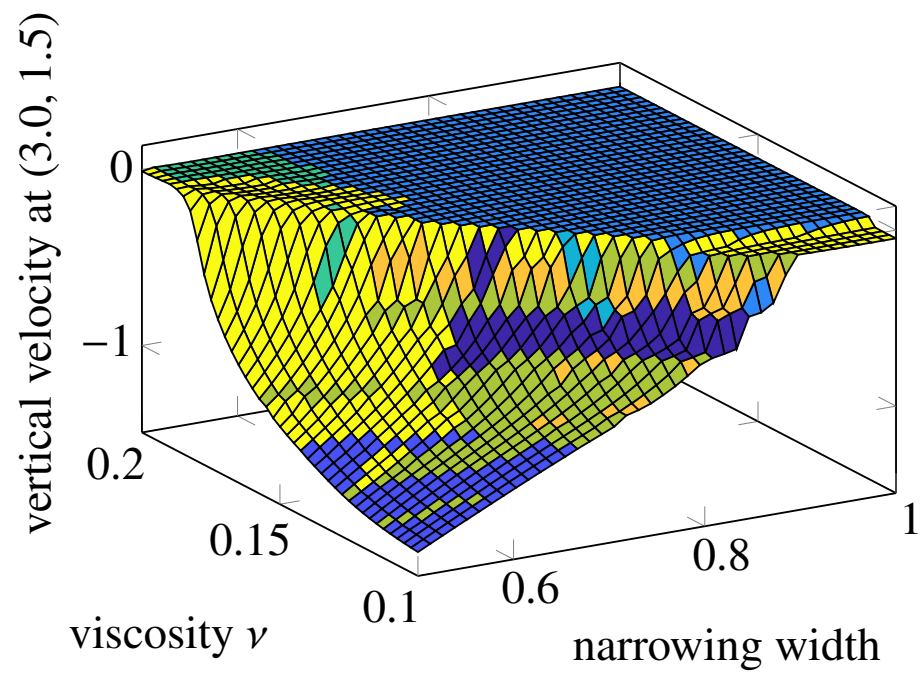

FIG. 3.7. Optimal cluster selection. Once again, different colors are used for different clusters.

cannot be generalized.

We consider test $3(10 \times 11$ sampling grid, overlapping clusters) to assess two variants of the regression ANN, which differ in how the training is done. One variant takes all clusters into account simultaneously and is called "regression ANN". It generates a mapping from $\mathbb{R}^{2} \mapsto \mathbb{R}^{8}$, i.e., the two dimensional parameter domain to the expected errors of the clusters. The second variant treats each clusters independently and is called "regression ANN, independent local ROMs". It generates eight mappings from $\mathbb{R}^{2} \mapsto \mathbb{R}$, i.e., one for each cluster. Table 3.3 reports the mean relative $L^{2}$ and $L^{\infty}$ errors for the velocity. Interestingly, taking all clusters into account simultaneously ("regression ANN") is about $10 \%$ more accurate than considering each cluster separately ("regression ANN, independent local ROMs"). Moreover, we observe that the mapping snapshot location to errors of local ROMs holds useful information and the ANN consistently gets closest to the optimum. Table 3.3 reports also the errors obtained with the Kriging DACE software* and with simply taking the cluster, which best approximates the closest snapshot. The closest snapshot is determined in parameter domain with the Euclidean norm. This is an easily implementable tool, which still

${ }^{*}$ Kriging DACE - Design and Analysis of Computer Experiments, http://www. omicron.dk/dace. html. 
performs better than the distance to the parameter centroid; see [7]. From Table 3.3, we see that this simple criterion performs only $15 \%$ to $20 \%$ worse and is very cheap to evaluate.

TABLE 3.3

Local ROM with different basis selection: mean relative $L^{2}$ and $L^{\infty}$ errors for the velocity over all reference parameter points; 1640 on a uniform $40 \times 41$ grid.

\begin{tabular}{lcc} 
& mean $L^{2}$ error & mean $L^{\infty}$ error \\
\hline optimum & 0.0048 & 0.0045 \\
regression ANN & 0.0068 & 0.0064 \\
regression ANN, independent local ROMs & 0.0076 & 0.0071 \\
Kriging DACE & 0.0077 & 0.0073 \\
distance to next best-approx. snapshot & 0.0081 & 0.0077
\end{tabular}

3.2. Comparing the global ROM, local ROM, and POD-NN approaches. In the previous section, we learned that the regression ANN criterion outperforms all other local basis selection criteria. In this section, we compare the local ROM approach with the regression ANN criterion to our global ROM approach and the POD-NN over the reconstruction of the bifurcation diagram.

Table 3.2 reports the mean relative $L^{2}$ and $L^{\infty}$ errors for the velocity for the three approaches under consideration. We consider four different numbers of samples: 42, 72, 110, and 240. It can be observed that the global ROM shows a slow convergence, not even $7 \%$ accuracy is reached with the finest sampling grid. The local ROM with regression ANN cluster selection shows no distinctive convergence behavior, which might indicate that the accuracy saturates at lower snapshot grid sizes. The POD-NN shows the fastest convergence, reaching about $0.3 \%$ error with the finest sampling grid. We note that the POD-NN training did not take overfitting into account. Overfitting occurs when the training data is more accurately approximated than the actual data of interest. It can be checked with having a validation set, whose accuracy is measured independently and not included in the training. Training can be stopped when the training data is more accurately approximated than the validation set.

TABLE 3.4

Comparison of global ROM, local ROM and POD-NN for four snapshot grids.

\begin{tabular}{llcc} 
& & mean $L^{2}$ error & mean $L^{\infty}$ error \\
\hline \multirow{2}{*}{42 snapshots } & global ROM & 3.7022 & 3.1120 \\
& local ROM + regression ANN & 0.0510 & 0.0486 \\
& POD-NN & 0.0108 & 0.0104 \\
\hline 72 snapshots & global ROM & 0.6970 & 0.5831 \\
& local ROM + regression ANN & 0.0103 & 0.0098 \\
& POD-NN & 0.0080 & 0.0075 \\
\hline \multirow{2}{*}{110 snapshots } & global ROM & 0.1044 & 0.0948 \\
& local ROM + regression ANN & 0.0068 & 0.0064 \\
& POD-NN & 0.0059 & 0.0053 \\
\hline \multirow{2}{*}{40 snapshots } & global ROM & 0.0762 & 0.0734 \\
& local ROM + regression ANN & 0.0101 & 0.0096 \\
& POD-NN & 0.0032 & 0.0027
\end{tabular}


Several remarks are in order.

REMARK 3.1. The data reported in Table 3.2 concerning the local ROM with regression ANN cluster selection and the POD-NN are sensitive to the neural network training. In addition, the data for local ROM approach are sensitive to the POD tolerances are changed. Nonetheless, Table 3.2 provides a general indication of the performance of each method in relation to the other two.

REMARK 3.2. A rigorous comparison in term of computational times is not possible because the different methods are implemented in different platforms. However, we can make some general comments. The POD-NN method has a significant advantage in terms of computational time: one does not need to assemble the trilinear reduced form associated to the convective term, which is our simulations takes about 1-3 hours. The time required for the POD-NN evaluation in the online phase is virtually zero, while projection methods need to do a few iterations of the reduced fixed point scheme. That takes about 10-30 s. On the other hand, the POD-NN required the training of the ANN. However, in our simulations that takes only about 20 minutes.

REMARK 3.3. We also investigated a local POD-NN, i.e., we combined a k-means based localization approach with the POD-NN. However, this led to significantly larger errors than the global POD-NN method. In general, the error of a local POD-NN approach was in the range of the error of the "local ROM + regression ANN". Thus, we did not pursue this approach any further.

4. Concluding remarks. We focused on reduced-order models (ROMs) for PDE problems that exhibit a bifurcation when more than one parameter is varied. For a particular fluid problem that features a supercritical pitchfork bifurcation under variation of Reynolds number and geometry, we investigated projection-based local ROMs and compared them to an established global projection-based ROM as well as an emerging artificial neural network (ANN) based method called POD-NN. We showed that k-means based clustering, transition regions, cluster-selection criteria based on best-approximating clusters and ANNs gain more than an order of magnitude in accuracy over the global projection-based ROM. Upon examining the accuracy of POD-NN, it became obvious that the POD-NN provides consistently more accurate approximations than the local projection-based ROM. Nevertheless, the local projection-based ROM might be more amenable to the use of reduced-basis error estimators than the POD-NN. This could be the object of future work.

\section{REFERENCES}

[1] A. Ambrosetti And G. Prodi, A Primer of Nonlinear Analysis, Cambridge University Press, Cambridge, 1993.

[2] S. L. BRunton, J. H. Tu, I. BRIGHT, AND J. N. KutZ, Compressive sensing and low-rank libraries for classification of bifurcation regimes in nonlinear dynamical systems, SIAM J. Appl. Dyn. Syst., 13 (2014), pp. 1716-1732.

[3] D. DRIKAKIS, Bifurcation phenomena in incompressible sudden expansion flows, Phys. Fluids, 9 (1997), pp. 76-87.

[4] R. FEARn, T. Mullin, AND K. Cliffe, Nonlinear flow phenomena in a symmetric sudden expansion, J. Fluid Mech., 211 (1990), pp. 595-608.

[5] T. HAWA AND Z. RUSAK, The dynamics of a laminar flow in a symmetric channel with a sudden expansion, J. Fluid Mech., 436 (2001), pp. 283-320.

[6] H. Herrero, Y. Maday, and F. Pla, RB (Reduced Basis) for RB (Rayleigh-Bénard), Comput. Methods Appl. Mech. Engrg., 261/262 (2013), pp. 132-141.

[7] M. W. Hess, A. Alla, A. Quaini, G. Rozza, And M. Gunzburger, A localized reduced-order modeling approach for pdes with bifurcating solutions, Comput. Methods Appl. Mech. Engrg., 351 (2019), pp. 379-403. 
[8] M. W. Hess, A. QUAINI, AND G. RozZA, Reduced basis model order reduction for Navier-Stokes equations in domains with walls of varying curvature, Int. J. Comput. Fluid Dyn., 34 (2020), pp. 119-126.

[9] - A spectral element reduced basis method for Navier-Stokes equations with geometric variations, in Spectral and High Order Methods for Partial Differential Equations-ICOSAHOM 2018, S. J. Sherwin, D. Moxey, J. Peiró, P. E. Vincent, and C. Schwab, eds., Lect. Notes Comput. Sci. Eng., 134, Springer, Cham, 2020, pp. 561-571.

[10] M. W. HeSS AND G. RozZA, A spectral element reduced basis method in parametric CFD, in Numerical Mathematics and Advanced Applications-ENUMATH 201, F. Radu, K. Kumar, I. Berre, J. M. Nordbotten, and I. S. Pop, eds., Lect. Notes Comput. Sci. Eng., 126, Springer, Cham, 2019, pp. 693-701.

[11] J. S. HesthaVEN AND S. UBBIALI, Non-intrusive reduced order modeling of nonlinear problems using neural networks, J. Comput. Phys., 363 (2018), pp. 55-78.

[12] B. Kramer, P. Grover, P. Boufounos, S. Nabi, AND M. Benosman, Sparse sensing and DMD-based identification of flow regimes and bifurcations in complex flows, SIAM J. Appl. Dyn. Syst., 16 (2017), pp. 1164-1196.

[13] T. Lassila, A. Manzoni, A. Quarteroni, And G. Rozza, Model order reduction in fluid dynamics: challenges and perspectives, in Reduced Order Methods for Modeling and Computational Reduction, A. Quarteroni and G. Rozza, eds., MS\&A. Model. Simul. Appl., 9, Springer, Cham, 2014, pp. 235-273.

[14] S. MishRA AND K. JAYARAMAN, Asymmetric flows in planar symmetric channels with large expansion ratios, Internat. J. Numer. Methods. Fluids, 38 (2002), pp. 945-962.

[15] A. Noor, On making large nonlinear problems small, Comput. Methods Appl. Mech. Engrg., 34 (1982), pp. 955-985.

[16] - Recent advances and applications of reduction methods, Appl. Mech. Rev., 57 (1994), pp. 125-146.

[17] A. NOOR AND J. PETERs, Multiple-parameter reduced basis technique for bifurcation and post-buckling analyses of composite plates, Internat. J. Numer. Methods Engrg., 19 (1983), pp. 1783-1803.

[18] _ Recent advances in reduction methods for instability analysis of structures, Comput. \& Struct., 16 (1983), pp. 67-80.

[19] F. PICHI, A. QUAINI, AND G. RozZA, A reduced order modeling technique to study bifurcating phenomena: application to the Gross-Pitaevskii equation, SIAM J. Sci. Comput., 42 (2020), pp. B1115-B1135.

[20] F. PICHI AND G. RozZA, Reduced basis approaches for parametrized bifurcation problems held by non-linear von Kármán equations, J. Sci. Comput., 81 (2019), pp. 112-135.

[21] M. Pintore, F. Pichi, M. W. Hess, G. RozZA, AND C. CANUTO, Efficient computation of bifurcation diagrams with a deflated approach to reduced basis spectral element method, Adv. Comput. Math., 47 (2021), Art. 1, 39 pages.

[22] G. Pitton, A. Quaini, And G. RozZA, Computational reduction strategies for the detection of steady bifurcations in incompressible fluid-dynamics: applications to Coanda effect in cardiology, J. Comput. Phys., 344 (2017), pp. 534-557.

[23] G. PITTON AND G. ROZZA, On the application of reduced basis methods to bifurcation problems in incompressible fluid dynamics, J. Sci. Comput., 73 (2017), pp. 157-177.

[24] F. Pla, H. HERRERO, AND J. VEGA, A flexible symmetry-preserving Galerkin/POD reduced order model applied to a convective instability problem, Comput. \& Fluids, 119 (2015), pp. 162-175.

[25] I. Sobey AND P. DRAZIN, Bifurcations of two-dimensional channel flows, J. Fluid Mech., 171 (1986), pp. 263-287.

[26] F. TERRAGNi AND J. Vega, On the use of POD-based ROMs to analyze bifurcations in some dissipative systems, Phys. D, 241 (2012), pp. 1393-1405.

[27] M. YANO AND A. PATERA, A space-time variational approach to hydrodynamic stability theory, Proc. R. Soc. Lond. Ser. A Math. Phys. Eng. Sci., 469 (2013), Art. 20130036, 23 pages. 